\title{
Relationship Between Variables of pre- and post-Randomized Questions in Computer-based Tests
}

\author{
Beesung Kam $^{1}$ \\ ${ }^{1}$ Professor, Dept. Medical Education, Pusan National University, South Korea, beesung@pusan.ac.kr
}

\begin{abstract}
Cheating during assessments can be a result of poor physical arrangement of exam settings. In multiple-choice question of computer-based tests (CBT), shuffling items is used to deter students from copying answers from their colleagues. Randomizing questions and shuffling items in multiplechoice questions are meant to set a copying-disabled environment for students. This study aims to statistically verify that shuffling is suitable for preventing cheating using quantitative data. Since object awareness may compromise the effectiveness of a survey method, we used quantitative data from the students' selection of items without them being aware and to further avoid compromising the data. A total of 472 CBT data from two academic semester years of 2013 and 2016 were collected from first-year students enrolled in two exams of Cell Regulation Mechanism and Basic Human Structure. Participants data collected from test score, multiple-question selection number, and seat number. The question item number of each student was compared with the item selection of five nearby students seated in different positions. Item selection data from students enrolled in Basic Human Structure exam compared to five nearby colleagues showed a correlation coefficient of less than 0.8 , indicating that there were no cases of cheating confirmed. In the case of Cell Regulation Mechanism exam, by ignoring the front seat position, (considered limitation of this study) the measurement of comparing individual students to five nearby colleagues showed a correlation coefficient above 0.8 among 13 students ( $\mathrm{n}=13$ ), about $5.8 \%$ of total students, meaning $5 \%$ of students either cheat or selected items based on their knowledge. Practically, by simply looking at the monitor of a colleague, justifying cheating is difficult; however, the measurement of quantitative data produced by the students'selection of items showed that question-shuffling and item randomization reduce cheating among students. The results of this study is also intended to introduce the feeling of a safer environment by justifying that shuffling would deter cheating.
\end{abstract}

Keywords: Deterrence in CBT, Randomizing CBT, Shuffling Items in CBT, Cheating in Assessment

\section{Introduction}

The role of technology in society has led to the use of computer-based tests (CBT) instead of paperbased tests (PBT), which are both widely accepted and practical. Students are more comfortable taking CBTs rather than PBTs. Although the benefits of using computers for testing are numerous, measuring test reliability and content competency is essential and challenging[1].

Copying answers from colleagues by looking at the work of other students is considered as cheating. Throughout this study, we prefer to use the term "cheating" to provide clarity to the context. A study [2] showed that the prevention of cheating is essential as test validity is crucial to overall learning, especially in medical schools. The method of cheating varies by classroom; they include the use of hidden notes, and using cellphones to get extra help or to cheat. Although we exercised some

Received: October 25, 2020; 1st Review Result: December 14, 2020; 2nd Review Result: January 28, 2021 Accepted: February 26, 2021 
methods of deterrence such as restricting students from bringing phones into the classroom, restricting students from going to the restroom during examination sessions, providing the students with a computer-based note instead of paper sheets, and blocking computers to prevent them from surfing the internet[3], restricting them from cheating seemed challenging.

Based on psychological perspective, many individual factors have been predicted for cheating behaviors including age, gender, self-esteem, grade and personality. There exist other factors such as pressure or perhaps burnout, physical layout of class, atmosphere and severity of punishment[4]. Reasons for cheating in medical school is also not very clear. Most schools do not have records of cheating allegations. Response bias, a flaw inherent to survey design and risk factors for responders may result in underreporting of cheating. Pressure to earn good grade, inadequate understanding of the curricula and perhaps burnout may be the reason behind cheating in medical school.

A study on nursing examinations[5] reveals that $10 \%$ of students copy answers from their colleagues by looking at their work. In this study we will explain that by shuffling questions only $5.8 \%$ of students in Cell Regulation Mechanism exam have correlation coefficient data of above 0.8 and it may be interpreted as a cheating behavior. However, the reliability of an examination can depend on the environment settings so that students can tackle exams solely by using their own knowledge[6].

A study [7] showed that an examination for large classrooms is constrained by the space provided for each examinee (situational factor) so that it is not difficult for students to cheat during the session.

Research [8][9] shows that online examinations have higher scores than normal classroom examinations due to the lack of supervision and the open access to forbidden materials. The suggestion to reduce the time allocated for examinations seemed helpful. However, regarding deterrence, reducing allocated time is also uncertain.

A similar research by [10] showed that randomizing questions and shuffling items reduce expectations of cheating, which pays off well. Students work harder to earn the grade themselves rather than relying on the classroom environment, which enhances learning.

This study aims to investigate the measurement of test reliability in the sense that quantitative statistical data are being used instead of qualitative surveys to support the hypothesis. The impact of conducting experiments on subjects suggests that participants should not be made aware of the experiment. This is because participants would probably change their behavior if they thought they were participating in an investigation. However, avoiding such affection, we shift the usage of our data source from surveys to quantitative data collected from question item selection in which there is no way students feel or think that their selection might be used for an investigation over cheating. Students believed that their computer-based selection was used solely for scores and ranking statistics.

Implication of rapid technology advancement can create tendency toward acceptance of innovation and the belief that technology will be useful. Study at [4] reveals that technology has also created novel way to cheat. Some of these methods are including copy and paste, downloading internet source of knowledge, getting help from mobile phone to text, photography of test questions, take note and make audio to yourself a method similar to calling, wireless method of transferring text. Although various method of deterrence such as blockage of mouse or internet or background has been implemented, the problem of students cheating is not going to go away however awareness of industry to provide better solution and school to educate importance of honesty for future role players are essential and significance to deter cheating.

The purpose of this study is to statistically verify if shuffling is suitable for preventing cheating using quantitative data. Question of "is shuffling item in CBT test, suitable for preventing cheating" previously investigated using student survey. Object awareness may compromise the effectiveness of a survey. Therefore, we used quantitative data from the students' selection of items without their being aware of any ongoing investigation related to cheating and to further avoid compromising the data. 


\section{Method}

The following subsections describe methodology of this study.

\subsection{Research Tool}

A total of 472 CBT data from two academic semester years of 2013 and 2016 were collected from first-year students enrolled in two exams, Cell Regulation and Basic Human Structure Mechanism at Pusan National University School of Medicine. The students took the test in a classroom with 70 desks, each facilitated with two desktops and 21-inch-wide monitors at a distance of 50, 90, 90, 120, and $120 \mathrm{~cm}$ with neighboring monitor respectively. Data collected from the participants included the test score, multiple-question selection number, and seat number.

\subsection{Research Subjects}

Pre-randomization refers to the state that questions and question items viewed for students without being shuffled, and post-randomization referred to the state that all questions and question items were randomized for students in which individual student views items in shuffling mode.

Participants were verbally discouraged from cheating and warned of facing consequences in the event that they did cheat. The participants of the pre- and post-assessments were unaware of any ongoing study determining dishonesty analysis of any kind.

The students were aware of punishment in the event they were caught cheating. However, no case or punishment of cheating were reported.

Following the suggestions for examination security guideline research by [3][11] showed in preand post-assessment, students were checked before entering the exam room for not bringing in forbidden materials, any devices (e.g., USB, phone, electric watch, etc.). Students were forbidden from using the restroom during the examination session and provided with one unique id before starting the examination. Accessing forbidden information that is automatically banned by the CBT system. Only questions and question items were shuffled in the post-assessment.

\subsection{Research Procedure}

The level of difficulty for the two courses in pre- and post-assessment data were similar, measuring $67.85 \%$ and $63.16 \%$ for the Cell Regulation Mechanism exam and $53.63 \%$ and $51.16 \%$ for the exam, Basic Human Structure in pre- and post-assessment, respectively.

The question item number of each student was compared with the item selection of five nearby students seated on the following sides: L: left side, LF: diagonal left-front with distance, F: front, RF: diagonal right-front with distance, and R: right side. The readability of the colleague's monitor for positions other than those mentioned above was significantly less for someone with normal vision; therefore, only five seating positions were selected for this experiment. For fairness of comparison, data with regard to the front and side rows in the classroom were eliminated, as practically, cheating in those positions was impossible. Data of 4 students (seated in the last row in classroom) of the Cell Regulation Mechanism exam in the pre-assessment were eliminated as the number of students in the post-assessment was less, and the participation rates were $100 \%$ and $98 \%$ for the pre- and postrandomization, respectively. Figure 1 illustrates the design of the study.

To simplify data procedure in case of pre-randomization two data sets of one-student and five-seats were collected separately. Correlation coefficient of two data sets were calculated to be used for prerandomization statistics. Same method also used for collecting post-randomization statistics. 


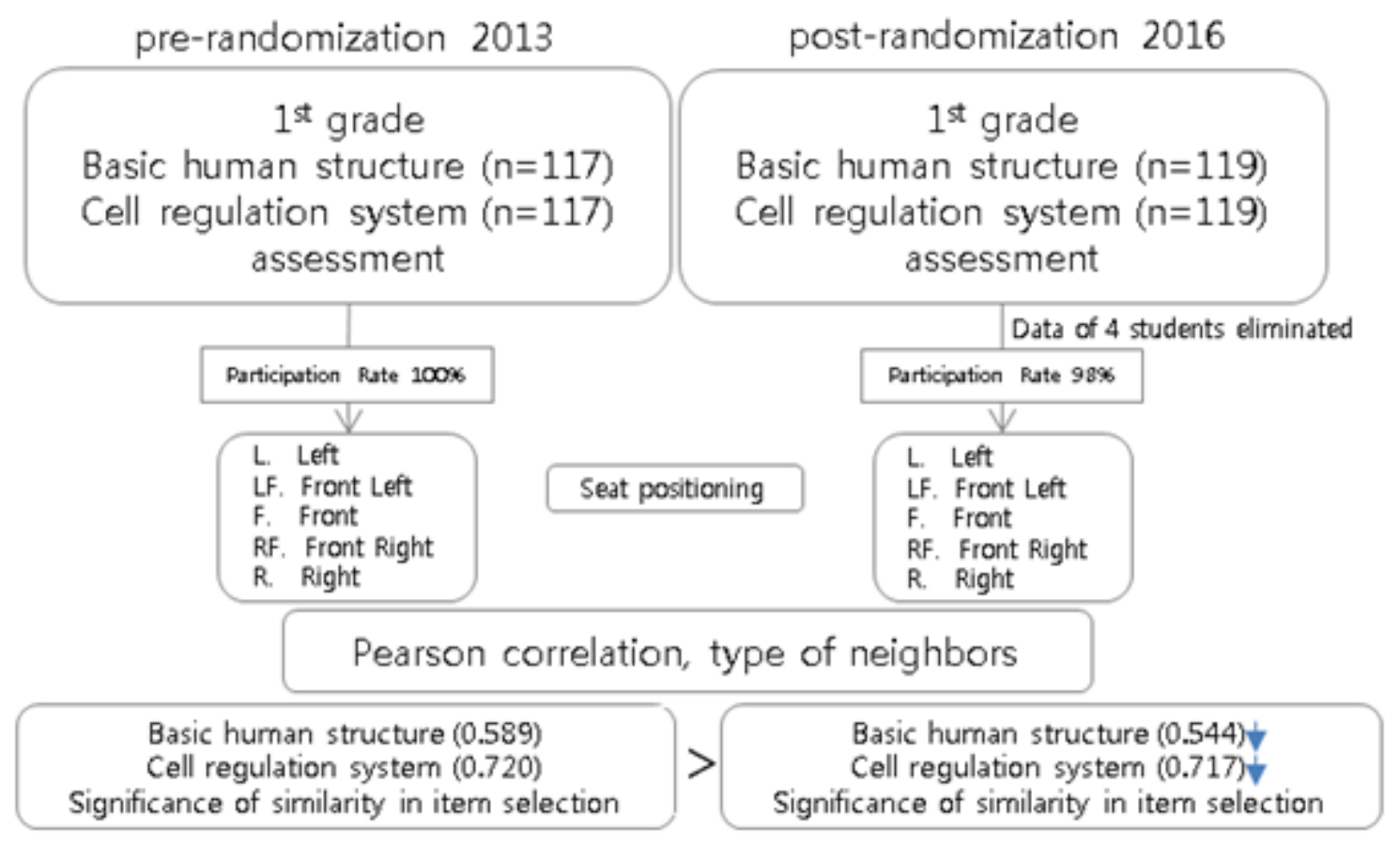

[Fig. 1] Illustration of the Enrollment and Participant Design

\subsection{Data Analysis}

The selected number of multiple-choice items in the dataset is separately compared with the five seating positions (L, LF, F, RF, and R) for the Pearson coefficient correlation for each type of the neighbors' analysis and presented as the average values of the pre- and post-assessments $(n=117)$ and $(\mathrm{n}=119)$.

The statistical significance was measured based on a correlation coefficient of 0.8 . Values less than 0.8 can be interpreted as case of no cheating, while value more than 0.8 can be interpreted as either cheating or in some case item selection based on the students' knowledge.

\section{Results}

This study focused on investigating the seating positions that enabled cheating. The seating positions were categorized as follows: L: left side, LF: diagonal left-front with distance, F: front, RF: diagonal right-front with distance, and R: right side. Assessment data were gathered in school after the course examination of pre- and post-question randomization with respect to year. Table 1. illustrates a comparison of the average correlation between student and the five different seating positions presented in pre- and post-randomization in two CBT exams.

In the left position (L), the total average of correlation with four other seating values reduced from 0.588 to .544 in the Cell Regulation Mechanism exam. The same position showed a reduction of average of correlation from .720 to .718 for the Basic Human Structure exam. The reduction differences showed a significant value of .04 and .002 , respectively.

In the left-front (LF) position, the average of correlation with four other seating values reduced from 0.587 to .543 in the Cell Regulation Mechanism exam. The same position showed a reduction of average of correlation from .725 to .717 for the Basic Human Structure exam. Reduction differences showed a significant value of .04 and .008 , respectively. 
[Table 1] Average of Correlations of Item Selections and Key Seating Positions

\begin{tabular}{ccc|cc} 
Seat Positions & \multicolumn{2}{c}{ Cell Regulation Mechanism } & \multicolumn{2}{c}{ Basic Human Structure } \\
\hline $\mathrm{N}=177, \mathrm{p}<0.001$ & Pre & Post & Pre & Post \\
\hline L (left) & $(2013)$ & $(2016)$ & $(2013)$ & $(2016)$ \\
LF (Left-Front) & .588 & $.544 \downarrow$ & .720 & $.718 \downarrow$ \\
F (Front) & .587 & $.543 \downarrow$ & .725 & $.717 \downarrow$ \\
RF (Right-Front) & .591 & $.540 \downarrow$ & .714 & $.719 \uparrow$ \\
R (Right) & .592 & $.550 \downarrow$ & .721 & $.717 \downarrow$ \\
& & $.543 \downarrow$ & .721 & $.719 \downarrow$
\end{tabular}

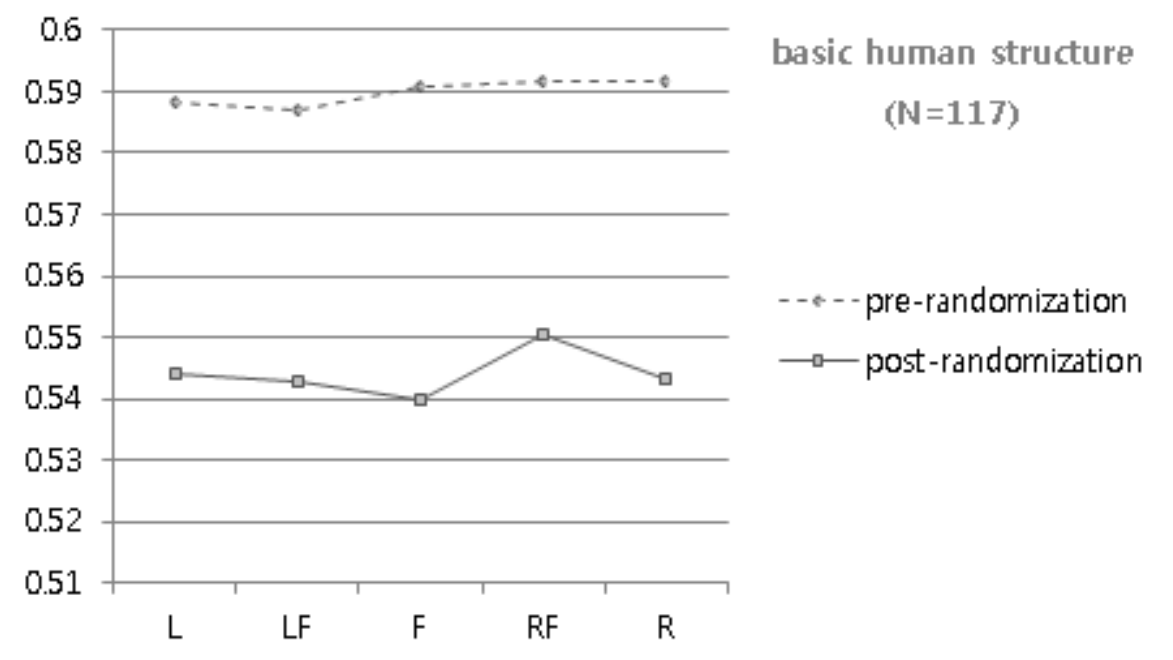

[Fig. 2] Illustration of Average Values in Correlation between Item Selection of Students in pre- and postRandomization

In the front position $(\mathrm{F})$, the average of correlation with four other seating values reduced from 0.591 to .540 in the Cell Regulation Mechanism exam (decreased value of .051) The same position for the Basic Human Structure exam showed an increasing value of average of correlation from .714 to .719 (increased value of .002; reason of increment explained in the limitation section) for the Basic Human Structure exam.

In the right-front $(\mathrm{RF})$ position, the average of correlation with four other seating values reduced from 0.592 to .555 in the exam, Cell Regulation Mechanism. The same position showed a reduction of average of correlation from .721 to .717 for the Basic Human Structure exam. The reduction differences showed a significant value of .037 and .004 , respectively.

In the right-front position (R), the average of correlation with four other seating values reduced from 0.592 to .543 in the Cell Regulation Mechanism exam. The same position showed a reduction of average of correlation from .721 to .719 for the Basic Human Structure exam. The reduction differences showed a significant value of .049 and .002 , respectively. 


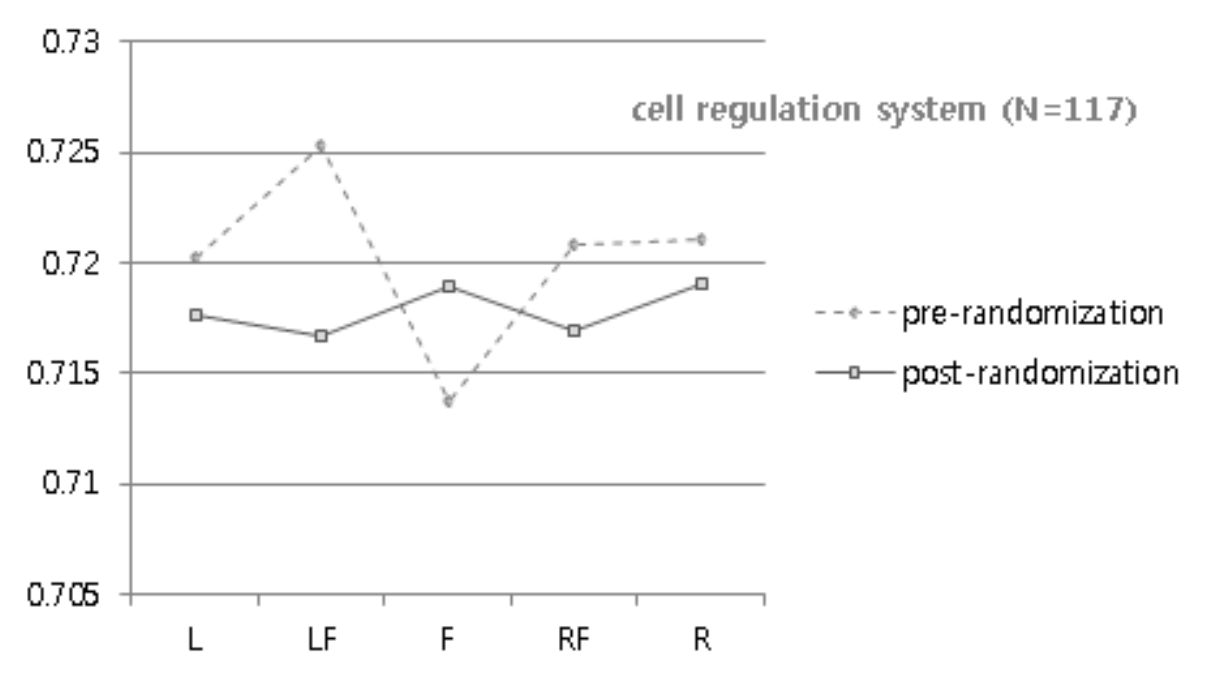

[Fig. 3] Illustration of Average Values in Correlation between the Item selection of Students in pre- and post- Randomization

The results showed that in every seating position, cases of copying answers from neighboring students were reduced after randomizing questions and question items except for the front seating position of the Cell Regulation Mechanism exam. Figures 2 and 3 illustrate the average of correlation of item selection of students by cheating from different seating positions.

\section{Conclusion}

Purpose of this study was to statistically verify that shuffling is suitable for preventing cheating using quantitative data.

Data collection of studies aim to reduce cheating are mostly based on survey data. Research [12] conducted using questionnaire and showed that due to lack of space provided for examination in large class sizes, finding students to cheat is not difficult. In case of e-assessment issues such as security and copying from web materials were a major concern. They concluded that cheating from colleagues may be a result of the physical arrangement of the exam setting.

An experiment in Dental Education suggested that a physical barrier would deter cheating. Research by [13] using survey data showed that covering monitors with screen filters increases their acceptability $90 \%$ among students, and helps students feel safer while writing exams. It was not clear whether they used randomization methodology, however, it is obvious that using screen filters darkens viewable content by reducing the readability of colorful pathology images. On the other hand, cost implication drawbacks which include the maintenance of filters, disassembly and storage, unwanted light reflections, and difficulty of navigating command lines, are also ignored. Another observation was that most of the students in medical school wear eyeglasses. Therefore, screen filters surely affect readability for students.

On the issue related to cheating research [5], it was suggested that $10 \%$ of the students copy answers from the work of other colleagues. Although reasons for cheating is not clear in medical school, survey data may compromise validity of content so that implication of such number may vary. Our data suggests in case of Basic Human Structure exam, because correlation coefficient values were all under 0.8 , we can interpret of no relation between selected items and seating positions. Therefore, no cases of cheating were observed. In the case of Cell Regulation Mechanism exam, the correlation coefficient value above 0.8 were approximately $5.8 \%$ of the students $(n=17)$. We can conclude that $5 \%$ of the students either cheated or selected items based on their knowledge. 
Medical students are under great pressure due to the competitive nature of the field. The adverse effect of this pressure is to frequently compare their work with that of others. The effectiveness of our system on students is not only to reduce cheating but also to reinforce the idea of a safe environment that reduces stress among students toward a fair environment for testing[2].

Research [14][15] shows that approximately 5\% of medical students cheat during the early years of medical school. The students mostly believed that cheating occurred. However, they lacked any clear consensus on how to act in the event they saw or felt that there were any cases of cheating by their colleagues. Students have different motivations to cheat. Some of these reasons include to get better grades, to appear proficient before their parents, to pass the course, and situational factors. Further, the majority never justified cheating. Although this system is meant to ensure that other students do not cheat, further study is needed to pursue the psychological perspective about why students go to such extents to cheat.

Figure 4 and 5 illustrates value of correlation coefficient of student compared to nearby seating positions in two exams of Basic Human Structure and Cell Regulation Mechanism. In one glance there is no different between post and pre randomization but in a closer look, more scatted with lower coefficient values appear. Even though the values make not much difference, comparing average values of coefficient makes more sense which have described in result of this study.

Shuffling questions and randomizing items are valid methods that can ensure reliable and fare CBT assessments in large classrooms. A copy disabling examination environment is one form of deterrence method. By this method, students work harder to earn the grade by themselves rather than relying on the classroom environment, which enhances learning. Although the idea of this study seems very old, the motivation of this study is to ensure a safer environment using quantitative data.

Collecting survey data from questionnaires is the most common methodology used in studying cheating behavior[16][17]; however, since students hide their dishonest behaviors, different supportive method is needed. This study measured quantitative data from pre- and post-examination in which the feelings of the students did not affect statistical analysis.

Although our study is based on quantitative data, our findings basically present rigorous statistics. We regulate our findings by relying on supervisor interviews for the final outcome; however, comparing quantitative results with qualitative interviews might seem like a mixed method and finding similar studies for comparison is very difficult.

On the other hand, rapid technological advancement can create acceptance of innovations and the belief that technology can be useful. Although our findings present rigorous statistics, more quantitative approaches needed to verify that rapid technology is reliable and safe for students' exam[18].

Attempting a question item selection through "guessing" rather than cheating can be mentioned as a bias. Further research is needed to verify such biased results by measuring the timing of selected items for ascertaining the probability of "guessed" items.

Another limitation of this study is that sorting was enabled during pre-examination depending on different self-selected groups. The effect of a particular factor may alter the overall results. Although tests have been conducted on different groups, external validity should be more of a concern in future researchers.

We recommend more studies using quantitative data from examination room.

Quantitative data including Item selection sequence, time of question selection, duration of time spent on each question in combine with value of question difficulty and standard deviation that can help understanding student behavior during examination. 


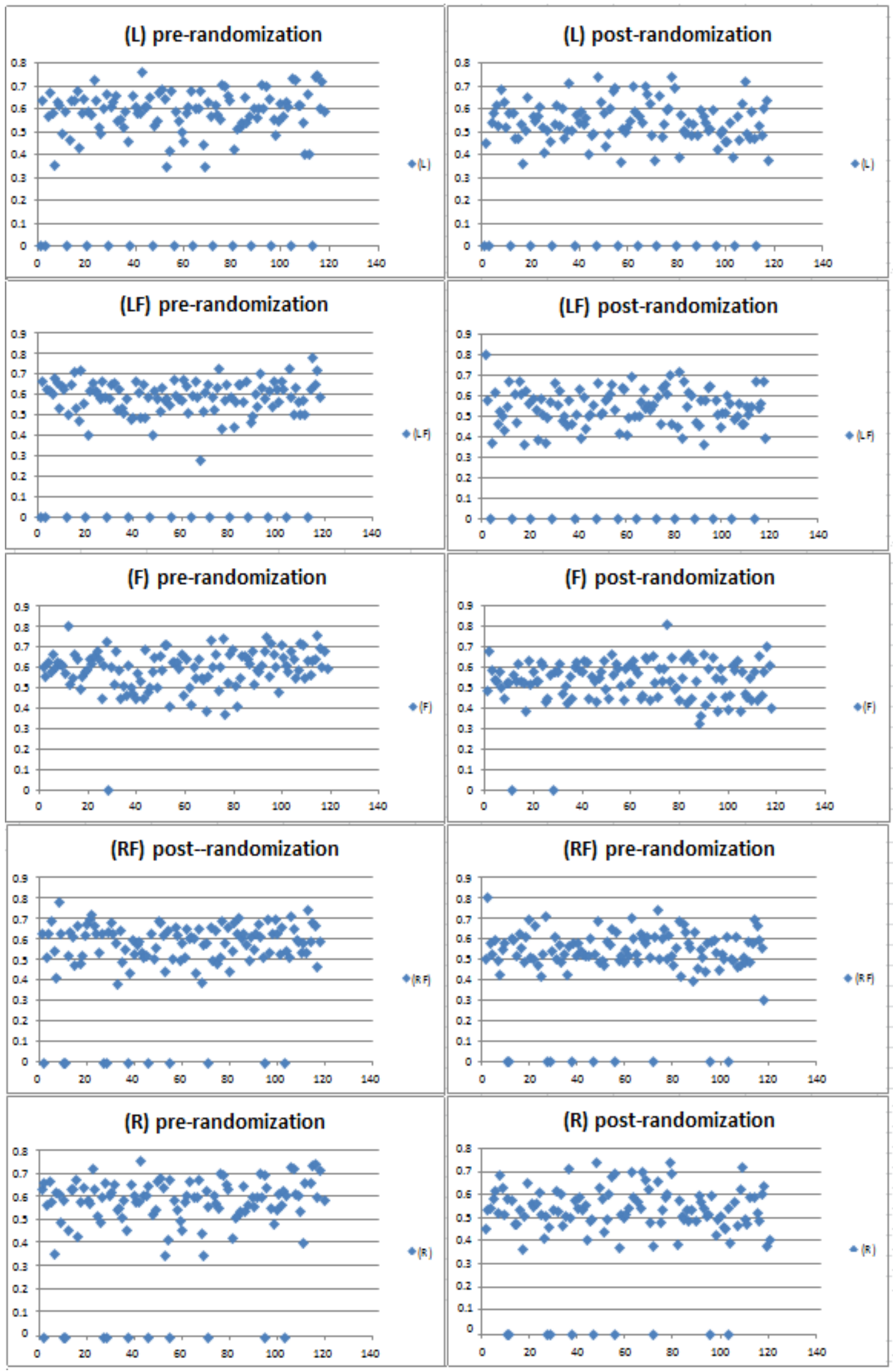

[Fig. 4] Illustration of Correlation Values between each Student and their Five Seating Positions (Basic Human Structure exam) 


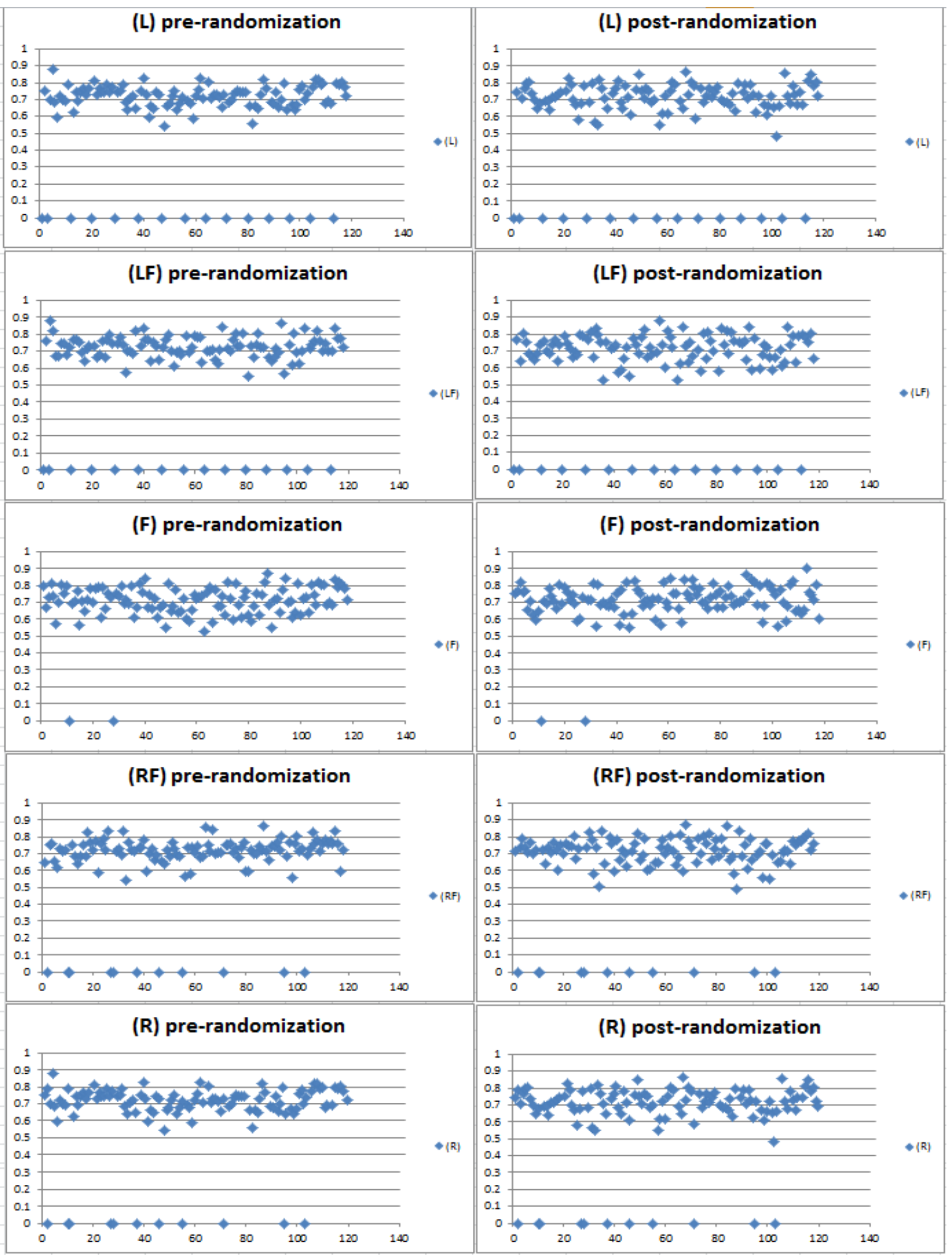

[Fig. 5] Illustration of Correlation Values between each Student and their Five Seating Positions (Cell Regulation Mechanism exam)

\section{Acknowledgments}

This work was supported by a clinical research grant in 2019 from Pusan National University Hospital. 


\section{References}

[1] P. Houdek, A Perspective on Research on Dishonesty: Limited External Validity Due to the Lack of Possibility of Self-Selection in Experimental Designs, Journal of Frontiers in Psychology, (2017), Vol.8, p.1566, https://doi.org/10.3389/fpsyg.2017.01566

[2] K. D. Royal, M. W. Hedgpeth, J. R. Mulkey, J. Fremer, The "10 Most Wanted" test cheaters in medical education, Med Educ Journal, (2016), Vol.50, No.12, pp.1241-1244, DOI: 10.1111/medu.13096

[3] S. M. Al-Saleem, H. Ullah, Security Considerations and Recommendations in Computer-Based Testing, Sci World Journal, (2014), Vol.7, http://dx.doi.org/10.1155/2014/562787

[4] J. O. Karl, J. Reid, R. Bartlett, Cyber Cheating in a Information Technology Age, Digithum, (2008), No.10, pp.19-28.

[5] J. L. Palmer, M. Bultas, R. L. Davis, A. D. Schmuke, J. B. Fender, Nursing Examinations: Promotion of Integrity and Prevention of Cheating, Nurse Educ, (2016), Vol.41, No.4, pp.180-184, DOI:10.1097/NNE.0000000000000238

[6] J. Dermo, Developing spaces for e-assessment: cases from the University of Bradford, Transforming Assessment webinar, (2012)

[7] Y. Miller, R. Izsak, Students' Involvement in Academic Dishonesty and Their Attitudes towards Copying in Exams and Academic Papers, Sociology and Anthropology, (2017), Vol.5, No.3, pp.225-232, DOI: 10.13189/sa.2017.050306

[8] D. Varble. Reducing Cheating Opportunities in Online Test, Atlantic Marketing Journal, (2014), Vol.3, No.3, pp.131149.

[9] A. Domnich, D. Panatto, A. Signori, N. L. Bragazzi, M. L. Cristina, D. Amicizia, R. Gasparini, Uncontrolled WebBased Administration of Surveys on Factual Health-Related Knowledge: A Randomized Study of Untimed Versus Timed Quizzing, Journal of Med Internet Res, (2015), Vol.17, No.4, pp.94-104, DOI:10.2196/jmir.3734

[10] D. P. Sullivan, An Integrated Approach to Preempt Cheating on Asynchronous, Objective, Online Assessments in Graduate Business Classes, Online Learning, (2016), Vol.20, No.3, pp.195-209, DOI: http://dx.doi.org/10.24059/olj.v20i3.650

[11] G. R. Cluskey, C. R. Ehlen, M. H. Raiborn, Thwarting Online Exam Cheating Without Proctor Supervision, Journal of Academic and Business Ethics, (2011), Vol.4.

[12] H. Mellar, R. Peytcheva-Forsyth, S. Kocdar, A. Karadeniz, B. Yovkova, Addressing Cheating in e-Assessment Using Student Authentication and Authorship Checking Systems: Teachers' Perspectives, International Journal of Education Integrity, (2018), Vol.14, No.2, pp.1-21, https://doi.org/10.1007/s40979-018-0025-x

[13] M. Escudier, J. Tricio, E. Odell, Student Acceptability of High-Stakes e-Assessment in Dental Education: Using Privacy Screen Filters to Control Cheating, Journal of Dental Education, (2014), Vol.78, No.4, pp.558-566, DOI:10.1002/J.0022-0337.2014.78.4.TB05706.X

[14] T. McClelland, J. Cuevas, A Comparision of Computer Based Testing and Paper Based Testing in Mathematics Assessment, The Online Journal of New Horizons in Education, (2020), Vol.10, No.2, pp.78-88

[15] H. Abdulghani, S. Haque, Y. Almusalam, S. Alanezi, Y. A. Alsulaiman, M. Irshad, S. Shaik, N. Khamis, SelfReported Cheating Among Medical Students: An Alarming Finding in a Cross-Sectional Study from Saudi Arabia, PLoS ONE, (2018), Vol.13, No.3, pp.1-14, https://doi.org/10.1371/journal.pone.0194963

[16] M. Isakov, A. Tripathy, Behavioral correlates of cheating: Environmental specificity and reward expectation, PLoS One, (2017), Vol.12, No.10, pp.1-12, https://doi.org/10.1371/journal.pone.0186054

[17] N. B. Kayisoglu, C. Temel, An Examination of Attitudes towards Cheating in Exams by Physical Education and Sports High School Students, Universal Journal of Educational Research, (2017), Vol.5, No.8, pp.1396-1402, DOI: 10.13189/ujer.2017.050813

[18] S. M. Glick, Cheating At Medical School: Schools Need A Culture That Simply Makes Dishonest Behaviour Unacceptable, British Medical Journal, (2001), Vol.322, No.7281, pp.250-251, https://www.jstor.org/stable/25466061 